\title{
MedienPädagogik
}

Zeitschrift für Theorie und Praxis der Medienbildung

www.medienpaed.com

ISSN 1424-3636

Jahrbuch Medienpädagogik 15:

Erziehungswissenschaftliche und medienpädagogische Online-Forschung:

Herausforderungen und Perspektiven

Herausgegeben von Johannes Fromme, Stefan Iske, Therese Leik, Steffi

Rehfeld, Jasmin Bastian, Manuela Pietraß und Klaus Rummler

\section{Möglichkeitsräume für bildende Begegnungen im Internet gestalten}

\section{Medienpädagogische Perspektiven für Soziale Netzwerke am Beispiel einer Online-Lernumgebung}

Tobias Hölterhof

\section{Zusammenfassung}

Soziale Netzwerke sind für die Medienpädagogik äusserst interessante Plattformen und Dienste im Internet, die Kontakte und Relationen von Nutzenden ermöglichen und abbilden. Neben der Analyse solcher Plattformen rückt ihre Gestaltung in den Fokus medienpädagogischer Forschung, wenn Online-Umgebungen zur Unterstützung pädagogischen Handelns eingesetzt werden. Doch wie können solche Plattformen als Bildungsumgebungen fruchtbar gestaltet werden? Was sind allgemeine Perspektiven für den Einsatz solcher Systeme? Der Beitrag entwickelt aus der von Otto F. Bollnow vorgelegten bildungs- und existenzphilosophischen Erörterung von Begegnungen eine Bildungsperspektive für Soziale Netzwerke. Diese Perspektive versteht Soziale Netzwerke als Möglichkeitsräume für Begegnungen: Sie strukturieren soziale Relationen und bieten Kommunikationswerkzeuge sowie Wahlmöglichkeiten. Doch hierfür müssen Soziale Netzwerke entsprechend gestaltet sein. Im Schnittfeld von Medienpädagogik und Webtechnologie stellt der Beitrag Ansätze für die prototypische Gestaltung solcher Plattformen aus einem modularen Content Management System vor. Er diskutiert Eigenheiten und Spezifika eines solchen 
Gestaltungsprozesses, welcher im Sinne dieser Bildungsperspektive das Aufspannen eines sozialen Möglichkeitsraumes fokussiert um Soziale Netzwerke zu Orten der Begegnung werden zu lassen.

\title{
Creating Opportunities for Encounters on the Internet: Media Educational Perspectives for Social Networks as exemplified by an online-learning Environment
}

\begin{abstract}
Social network sites are interesting online platforms for education, enabling contacts and relations of users. Beside analysing those platforms, when they are used to support pedagogical action their design moves into the focus of educational research. But how to design social network sites fruitful as educational environments? What are general perspectives for using such systems? This article develops an educational perspective for social networks sites referring to Bildung as a general educational issue. It draws on the philosophical discussion of encounter as Bildung presented by Otto F. Bollnow. Following this perspective, social networks sites may be framed as spaces of possibilities for encounters: they structure social relationships, offer options as well as communication tools to realise those options. But therefore social network sites must be designed accordingly. In the intersection of education, media and web technology the article presents approaches for prototyping such platforms using a modular content management system. It discusses characteristics of a design process following the mentioned educational perspective and focussing on the development of a space of possibilities for encounter in order to foster social network sites as a place of encounter.
\end{abstract}




\section{Soziale Netzwerke als Gegenstand medienpäda- gogischer Gestaltung und Analyse}

Als Soziale Netzwerke werden zwei verschiedene Phänomene bezeichnet, die beide medienpädagogisch von Bedeutung sind. $\mathrm{Zu}-$ nächst handelt es sich im Kontext des Internets um spezifische Plattformen und Dienste, die soziale Relationen unter Teilnehmenden abbilden und entsprechend dieser Relationen eine individualisierte Online-Umgebung bereitstellen. Solche Plattformen werden auch als «Social Network Sites» bezeichnet (vgl. boyd und Ellison 2007). Diese Plattformen werden aus unterschiedlichen Perspektiven wie etwa Medienerziehung, Medienkompetenz oder Medienbildung im Kontext pädagogischen Forschens und Handelns relevant (vgl. Hölterhof 2017b; Jörissen und Marotzki 2009; Meder 2007b; Pietraß 2009). Andererseits werden im Kontext der Soziologie auch soziale Relationen oder Verbindungen zwischen Menschen ganz allgemein als soziale Netzwerke bezeichnet, wie etwa Bekanntschaften oder Freundschaften. Insbesondere die soziale Netzwerkanalyse als sozialwissenschaftliche Forschungsmethode deckt solche Strukturen auf. Diese beiden Bezeichnungen sind theoretisch zwar verschieden, besitzen aber in der medienpädagogischen Gestaltung von OnlineUmgebungen eine gemeinsame Relevanz: Social Network Sites sind die Plattformen und Umgebungen im Internet, auf denen soziale Netzwerke im Sinne der Verbindung von Teilnehmenden stattfinden und gefördert werden können. Dabei rückt eine solche Vernetzung der Teilnehmenden über Online-Plattformen in den Fokus medienpädagogischen Handelns (vgl. Kerres et al. 2016).

Zwei Beispiele sollen die Verbindung von Online-Plattform und Vernetzung der Teilnehmenden als Gegenstand medienpädagogischer Gestaltung und Praxis verdeutlichen (siehe Abbildung 1). Die religionspädagogische Online-Plattform «rpi-virtuell» verbindet eine Datenbank frei verfügbarer Bildungsmedien mit einem Sozialen Netzwerk, welches es Teilnehmenden ermöglicht, ein eigenes Profil und eigene Gruppen anzulegen (vgl. Heinen et al. 2014). Das 
Angebot ist offen im Internet verfügbar, richtet sich insbesondere an Religionspädagoginnen sowie -pädagogen und verfolgt das Ziel, neben der Bereitstellung und Verlinkung von Inhalten auch eine Vernetzung der entsprechenden Akteure zu fördern. Ein anderes Beispiel stellt die Online-Lernumgebung «OnlineCampus» dar (vgl. Hölterhof 2018a; Kerres et al. 2011; Kerres und Rehm 2015). Sie dient der Durchführung von Online-Studiengängen und ist demnach eine geschlossene Plattform, welche den Teilnehmenden Kommunikations- und Kollaborationsmöglichkeiten zur Verfügung stellt. Ziel ist hier die Förderung von sozialen Lernprozessen über das Internet. Beide Plattformen bieten die für Soziale Netzwerke charakteristischen Eigenschaften, dass Nutzende hier eigene Profile anlegen und Relationen zu anderen Nutzenden herstellen können. Sie bilden damit soziale Strukturen ab und gestalten Umgebungen, in denen Informationen und Inhalte in einer sozialen Online-Plattform eingebettet werden (vgl. boyd und Ellison 2007; Röll 2010).

Diese Annäherungen an Soziale Netzwerke als Plattformen und Umgebungen im Internet sind geprägt durch eine gestaltungsorientierte Perspektive auf medienpädagogische Forschung (vgl. Kerres und de Witt 2011; Tulodziecki et al. 2013, 2014). Modelle und Erörterungen eines solchen Forschungsprozesses systematisieren den Bezug zur Praxis anhand der inkrementellen Entwicklung und Evaluation von Prototypen (vgl. Akker 2006; Easterday et al. 2014; Tulodziecki et al. 2013, 212; 2014, 13). Typische Phasen dieses Prozesses beinhalten zunächst eine Konzeption, dann eine prototypische Umsetzung bzw. Anwendung, Evaluation sowie schliesslich Revision und Überarbeitung. Ziel dieses Prozesses ist es, sowohl Theorieentwicklung zu fördern als auch den Prototypen durch die Iterationen zu verbessern. Der Prozess findet als Zusammenwirken oder Kooperation von Wissenschaftlern und Praktikern statt (vgl. Tulodziecki et al. 2014, 13). 

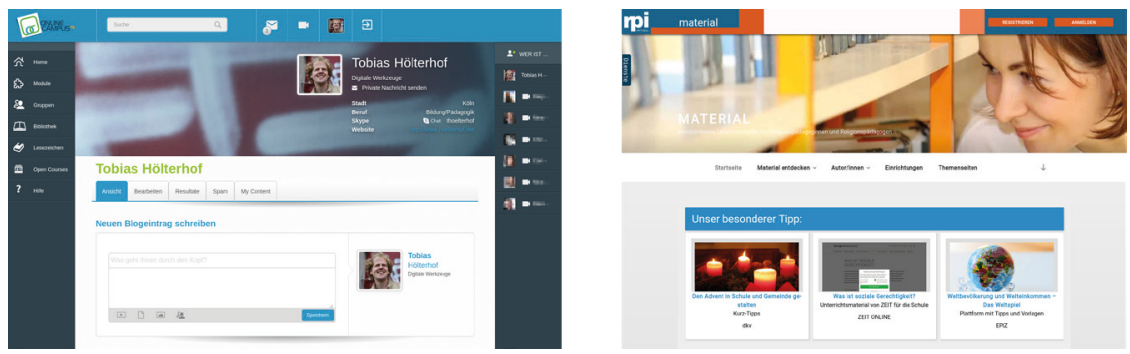

Abb. 1.: Zwei Beispiele für die medienpädagogische Gestaltung Sozialer Netzwerke im Internet: Links die Online-Lernumgebung «OnlineCampus», rechts die religionspädagogische Online-Plattform «rpi-virtuell.de». Quelle: eigene Screenshots.

Indem die derart entwickelten Angebote und Plattformen eine Vernetzung der Teilnehmenden ermöglichen und durch entsprechende Aktivierung die Kommunikation oder Kollaboration der Teilnehmenden gezielt fördern, entsteht eine Interaktionsstruktur, die anhand einer sozialen Netzwerkanalyse untersucht werden kann. Die Spuren sozialer Interaktionen sind in solchen Online-Umgebungen weniger flüchtig und werden oft langfristig in einer Datenbank gespeichert. In alltäglichen Situationen und ausserhalb von OnlinePlattformen sind solche Interaktionen oftmals schwerer zu erfassen und zu erforschen. Insbesondere in der Phase der Evaluation kann eine soziale Netzwerkanalyse Charakteristika der auf den Plattformen realisierten Interaktions- und Kommunikationsstruktur erforschbar machen, etwa hinsichtlich der Zentralität oder Konzeptration der Interaktion zentraler Personen und Akteure (vgl. Freeman 1978). Sofern die Vernetzung von Teilnehmenden als konzeptionelles Ziel medienpädagogischer Gestaltung fokussiert wird, können solche Netzwerkanalysen Rückschlüsse auf Gestaltungsprozesse zulassen.

Soziale Netzwerke bezeichnen im Kontext der Soziologie soziale Strukturen, die als gerichtete Beziehungen zwischen sozialen Entitäten gewissermassen den Gegenstandsbereich der Soziologie selbst darstellen (vgl. Rürup et al. 2015, 79). Als solches beschreiben sie im Kontext der Medienpädagogik die soziale Eingebundenheit des Handelns in und mit Medien. Eine wesentliche Eigenschaft der 
sozialen Netzwerkanalyse ist ihre Fokussierung auf Relationen. Während klassische sozialwissenschaftliche Forschung überwiegend Eigenschaften von Individuen erfassen, werden hier in einer Adjazenzmatrix die Nachbarschaften oder Beziehungen der Akteure dargestellt. Das soziale Netzwerk im Rahmen dieser Methode bezeichnet demnach eine Menge von Akteuren oder Knoten sowie deren Verbindungen untereinander (vgl. Hölterhof 2018b; Rürup et al. 2015, 21; Wasserman und Faust 1994, 9). Auf diese Weise können etwa Soziogramme als Graphen erstellt sowie die Positionen von Individuen in einer sozialen Struktur analysiert werden. Verbindungen stellen etwa Interaktionen von Teilnehmenden auf der Plattform dar, wie etwa das Antworten in einem Forum.

Die soziale Netzwerkanalyse als sozialwissenschaftliche Forschungsmethode gilt als theoretisch offen. Sie impliziert somit weder eine spezifische Theorie des Sozialen, noch einen Netzwerkbegriff, der Veränderungen in der menschlichen Sozialität konstatiert. Die grafentheoretische Beschreibung eines Netzwerks bildet die logische und mathematische Basis für die empirische Sozialstrukturanalyse, deren Ergebnisse jedoch in den Kontext einer Theorie eingeordnet werden müssen, um die sozialen Dynamiken verständlich werden zu lassen (vgl. Schweizer 1996, 112). Insofern stellt sich aus der Perspektive einer gestaltungsorientierten Bildungsforschung die Frage nach einem theoretischen Rahmen, aus dem heraus solche sozialen Analysen interpretiert und eingeordnet werden können. Der vorliegende Beitrag möchte aus einer bildungsphilosophischen Erörterung der Begegnung Aspekte eines solchen Rahmens ableiten, die insbesondere Soziale Netzwerke als Orte der Bildung fokussieren. 


\section{Begegnungen in Sozialen Netzwerken als Aspekt medienpädagogischer Konzeption}

Zur Einordnung des anthropologischen Phänomens der Begegnung in einen medienpädagogischen Kontext ist es zunächst gewinnbringend, sich diesem Phänomen aus einer bildungsphilosophischen Perspektive zu nähern. Da Medien schliesslich auch anthropologische Funktionen übernehmen, können dann Aspekte des Phänomens in den Bereich des Internets übertragen werden (vgl. Spanhel 2014). Dieses Vorgehen ermöglicht es auch, Konzeptions- und Gestaltungsprozesse als medienpädagogische Online-Forschung in einem bildungstheoretischen Rahmen zu verorten und hieraus Aspekte für die Gestaltung von Online-Plattformen abzuleiten. Einen solchen bildungstheoretischen Rahmen können etwa Krisen oder Kontingenzerfahrungen darstellen, die im Kontext transformatorischer Bildungstheorien untersucht werden (vgl. Koller 2011, 109f.). Diesen Erlebnissen werden prägende Erfahrungen zugeschrieben, die sich letztlich auch im Handeln der Personen manifestiert. Die pädagogische Bedeutung dieser existenziellen und krisenhaften Charakteristik des menschlichen Daseins wird von Otto F. Bollnow als unstetige Bildung konkretisiert. Er folgt dabei einem Ansatz, der pädagogische Impulse aus der Existenzphilosophie ableitet. Dabei stellt er einer stetigen Auffassung von Bildung im Sinne einer allmählichen Vervollkommnung unstetige Formen menschlichen Werdens entgegen, die Sprünge und Inkonsistenzen aufweisen (vgl. Bollnow 1977, 22; Koskela 2012, 54). Diese Unstetigkeit bezieht sich auf unvorhergesehene und spontane Veränderungen in einem Prozess, der jedoch gleichsam ein spezifisch menschliches Potenzial der Transformation darstellt.

Insbesondere erörtert Bollnow die Begegnung als eine solche unstetige Form der Bildung und enthüllt sowohl die Begegnung mit Menschen als auch die Begegnung mit Themen und Inhalten als sprunghafte, erschütternde und unvorhersehbare Momente, die ein Potenzial hin zu Engagement und Transformation beinhalten (vgl. 
Bollnow 1977, 99). Auf diese Weise rückt in der Begegnung etwas Fremdes und Wirklichkeitserweiterndes in die Aufmerksamkeit des Daseins und wird aus einem Blickwinkel erfahrbar, welcher zuvor noch nicht bekannt war. Obgleich Begegnung zunächst ein soziales Phänomen bezeichnet, nämlich die Begegnung von Menschen, so verweist es im Kontext dieser existenziellen Pädagogik auf eine spezifische Form der Berührung und der Betroffenheit, die als solche auch gegenüber Themen oder historischen Personen auftreten kann. Dieses Objekt der Begegnung ist durch ein «gegen» charakterisiert. Es beinhaltet demnach etwas Unabhängiges und Unbekanntes, das nicht selbstverständlich ist und demnach einen gewissen Widerstand besitzt. Eine Begegnung kann somit als Metapher verstanden werden, die einen inneren und existenziellen Prozess als räumliches Geschehen ausdrückt und dabei nicht nur die Begegnung mit Personen, sondern auch mit Themen oder Ideen bezeichnet (vgl. ebd., 97f.).

Das Unbekannte in der Begegnung muss jedoch zuvor erfahren werden. Insofern ist die Begegnung abhängig von Strukturen, die den Möglichkeitsraum für Begegnungen charakterisieren: Es muss zunächst die «Breite der Möglichkeiten» erfahren werden (Bollnow 1977, 124). Begegnung als spontaner, unplanbarer und unstetiger Prozess kann also nur in Bezug auf etwas stattfinden, was zuvor erlebt oder kennengelernt wurde. Handelt es sich um soziale Begegnungen mit anderen Menschen, so ist hier auch eine soziale Struktur angesprochen, die etwa durch soziale Netzwerkanalysen beschreibbar und empirisch untersuchbar wird. Insbesondere die Eigenheit computervermittelter Kommunikation, soziale Erfahrungen in der Reziprozität mehrerer Interaktionen zu ermöglichen, unterstreicht die Strukturgeprägtheit von Begegnungen in Online-Umgebungen. An die Stelle einer unmittelbaren und augenblicklichen Wahrnehmung einer anderen Person tritt demnach eine andauernde Progression mehrerer Interaktionen (vgl. Walther 1992, 80). Ein solcher Austausch hinterlässt Spuren in den Datenbanken der Systeme und formt gleichsam einen Möglichkeitsraum für Begegnungen. Insbesondere stellt Kommunikation und Zusammenarbeit hier eine 
anthropologische Funktion von Medien dar, die durch das Internet unterstützt werden kann. Insofern können Begegnungen mit Themen oder Personen durch das Internet unterstützt werden.

Neben der Strukturierung von Begegnungen durch den Möglichkeitsraum der vorhergehenden Erfahrung sowie durch das Objekt der Begegnung wird für Bollow eine Begegnung ferner durch die Unstetigkeit des Phänomens sowie durch das Engagement der involvierten Menschen konstituiert (vgl. ebd., 99f.). Die Unstetigkeit verweist auf eine Offenheit des Prozesses: er ist zwar strukturiert durch den Möglichkeitsraum vorhergehender Erfahrung, jedoch nicht hierdurch determiniert. Entsprechend kann auch die Existenzphilosophie als die philosophische Denkrichtung, der Bollnow in seinen Ausführungen zu einer existenziellen Pädagogik folgt, als eine Philosophie dargestellt werden, die das transformative Potenzial menschlichen Handelns und Erlebens betont (vgl. Hölterhof 2017b). Die Begegnung als existenzielles Phänomen mit Themen und Personen besitzt demnach eine Ergebnisoffenheit. Diese Offenheit liegt darin begründet, dass die Existenzphilosophie den Menschen in einem besonderen Mass ein Interesse und eine Freiheit in der Ausgestaltung ihrer Existenz zuschreibt. Ronald Hitzler sieht demnach für den Kontext soziologischer Forschung in der Existenzphilosophie eine theoretisch-praktische Strategie, einer Tendenz der «Verdinglichung des Gegen-Standes» entgegenzuwirken (Hitzler 1982, 154). Eine solche Verdinglichung kann etwa darin bestehen, die Lernenden oder Teilnehmenden in medienpädagogischen Konzeptionen und Analysen nicht als Subjekte und Handelnde zu begreifen.

Aus der Perspektive medienpädagogischer Gestaltung und Konzeption kann diese theoretisch-praktische Strategie der Existenzphilosophie fruchtbar gemacht werden, indem die sozialen Umgebungen mit einer gewissen Offenheit für eigengeleitete Prozesse gestaltet werden. Begegnungen sind demnach singuläre Geschehnisse und abhängig von der jeweiligen Situation und auch von der Annahme durch die Teilnehmenden selbst. Auch Soziale Netzwerke 
im Internet sind Orte spontanen und sozialen Handelns, die nicht gänzlich durch Instruktionen und Strukturen bestimmt sind, sondern Momente der Spontaneität und Unstetigkeit in der Begegnung mit neuen und unbekannten Themen und Personen beinhalten. Solche Momente zeichnen nach Otto F. Bollnow bildende Begegnung aus. Demnach verlangt eine Begegnung auch einen gewissen Einsatz vom Dasein: etwa als Interesse. Angesprochen ist hier die Bereitschaft des Daseins, sich auf eine Begegnung einzulassen und sich in ihr zu bewähren. Bollnow weist darauf hin, dass eine bildende Begegnung einen Menschen auf die Probe stellt, dass ein Dasein diese Begegnung in der Freiheit seiner Existenz annehmen muss (vgl. Bollnow 1977, 100). Diese Charakterisierung bildender Begegnungen zeigt den performativen Charakter der Existenz. Die menschliche Existenz ist demnach in ihrer konkreten Ausgestaltung ein durch das Dasein selbst geformtes Gebilde (vgl. Hölterhof 2017a, 204).

Zusammenfassend kann die Begegnung als existenzielles Phänomen nach Bollnow durch folgende Aspekte charakterisiert werden:

- Breite der Erfahrung: Diese steckt den Möglichkeitsraum für Begegnungen ab und strukturiert mögliche Begegnungen, ohne sie zu determinieren.

- Objekt der Begegnung: Gegenstände der Begegnung sind Themen, Menschen oder auch historische Personen, die in der Begegnung als unbekannt, fremd aber wirklichkeitserweiternd erlebt werden.

- Unstetigkeit des Begegnungsprozesses: Existenzielle Begegnungen besitzen eine Offenheit hinsichtlich der Gestalt, der Form und der Folgen dieser Begegnung für das erlebende Subjekt.

- Einsatz bzw. das Engagement des involvierten Subjekts: Eine Begegnung besitzt Momente des Ausprobierens, des Wagnis und stellt das Subjekt auf die Probe.

Das Phänomen einer bildenden Begegnung, die im Rahmen zuvor erfahrener Möglichkeiten stattfindet und das Potenzial besitzt, in einem spontanen Augenblick Erschütterung, 
Wirklichkeitserweiterung und Engagement einer dieser Möglichkeiten gegenüber zu entwickeln, beschreibt ein Erlebnis, welches auch in medialen Umgebungen handlungswirksam werden kann. Soziale Netzwerke als Online-Plattformen können Eindrücke und Erlebnisse von Menschen sowie von Themen vermitteln. Das Internet ist demnach eine Umgebung, die mannigfaltige Inhalte und Informationen zu Themen bereithält, die in Sozialen Netzwerken erlebt, mitgeteilt und eingebettet werden. Diese Aspekte zeigen, dass Soziale Netzwerke im Internet eine gewisse Eignung besitzen, solche Möglichkeitsräume aufzuspannen. Eine gestaltungsorientierte medienpädagogische Online-Forschung kann die Konzeption sozialer Netzwerke als solche Möglichkeitsräume für Begegnungen konkretisieren, erproben und weiterentwickeln.

\section{Gestaltung Sozialer Netzwerke im Internet als Möglichkeitsräume für Begegnung}

Medienpädagogische Online-Forschung als gestaltungsorientierte Bildungswissenschaft zu verstehen bedeutet, neben der Analyse Sozialer Netzwerke im Internet sich diesen Plattformen und Dienste auch als Gegenstand der Gestaltung anzunähern. Damit sind nicht nur technologische Gestaltungsdimensionen angesprochen, sondern auch die Umsetzung und Implementierung pädagogischer und didaktischer Strukturen, Anreize und Handlungsmöglichkeiten. Im Bezug auf die technologische Gestaltung kommt den Möglichkeiten und Grenzen der Webtechnologie ein besonderer Stellenwert zu, denn sie bestimmt Rahmenbedingungen der zu erprobenden Online-Umgebung. Die Verwendung und modulare Erweiterung bestehender Frameworks wie etwa Content Management-Systeme bietet insbesondere für Prototypen einen interessanten Kompromiss aus Breite der Gestaltungsfreiheit bei vergleichsweise geringer Komplexität des Entwicklungsprozesses hin zu einem Prototyp. Doch die Verfügbarkeit sozialer Kollaborations- und Kommunikationsangebote auf Online-Plattformen bedingt noch nicht deren Verwendung. 
Anreize und Motivationen sind weitere Gestaltungsdimensionen, die die Online-Umgebungen mit Leben füllen und Aktivitäten der Teilnehmenden begünstigen. Anhand der oben aufgeführten Charakteristika bildender Begegnungen sollen im Folgenden Gestaltungsimpulse für Soziale Netzwerke als Möglichkeitsräume für solche Begegnungen herausgearbeitet werden. Dabei wird die Konzeption und Umsetzung des «OnlineCampus» als Beispiel herangezogen, einer Lernumgebung für Online-Studienprogramme im Kontext wissenschaftlicher Weiterbildung an der Universität Duisburg-Essen.

Dass Online-Umgebungen zumindest Anteil an alltäglichen und authentischen Möglichkeitsräumen für Begegnungen mit Themen und Personen haben, wird im Kontext der Medienbildung erörtert (vgl. Hölterhof 2017b; Jörissen und Marotzki 2009; Meder 2007b; Pietraß 2009). Insbesondere kann hierbei hervorgehoben werden, dass das Internet als Kommunikations- und Informationsumgebung einen Zugang zu den oben genannten Gegenständen für Begegnungen darstellt. Auch die oben erwähnten Beispiele zeigen, dass Online-Umgebungen Möglichkeitsräume für Themen und Personen aufspannen. Insbesondere ist es ein Charakteristikum Sozialer Netzwerke im Internet, dass sie eine Vielfalt an Themen, Kontexten und Handlungen fokussieren (vgl. boyd und Ellison 2007). So gibt es Soziale Netzwerke für den Austausch von Fotos, Videos, Blogs, Nachrichten etc. Als Möglichkeitsraum für Begegnung ist diese Heterogenität interessant, denn diese stellt mögliche Gegenstände für Begegnungen dar.

Für die medienpädagogische Gestaltung eigener Sozialer Netzwerke als Plattformen im Internet kann diese Vielfältigkeit als Beispiel für Konzeptionen und Implementierungen dienen. Diesbezüglich ist für eine eigene Gestaltung zunächst herauszustellen, welche Formen und Typen von Inhalten und Informationen neben sozialen Kontakten als mögliche Gegenstände der Begegnung auf einer Plattform fokussiert werden. Im Kontext der Lernumgebungen «OnlineCampus» werden etwa neben Lerninhalten und Lernmaterialien, die 
im Kontext von Kursen und Modulen bereitgestellt werden, auch wissenschaftliche Artikel importiert. Diese Artikel dienen der Unterstützung eigengeleiteter und forschender Lernprozesse, wie sie für hochschulisches Lernen charakteristisch sind. Als Soziales Netzwerk sind diese Inhalte stets in den Kontext sozialer Relationen und Umgebungen eingebettet. So können sie etwa geteilt und kommentiert werden.

Für die technische Implementierungen eines solchen Sozialen Netzwerks bietet es sich an, modulare Systeme als Frameworks zu verwenden. So können etwa durch Erweiterung von Content oder Learning Management-Systemen viele Gestaltungskonzepte umgesetzt werden, ohne die Umgebung vollständig neu zu programmieren. Insbesondere stellen solche Frameworks eine Sammlung bereits programmierter und erprobter Erweiterungen zur Verfügung, die in Prototypen integriert werden können. Der «OnlineCampus» nutzt das Content Management-System Drupal in Kombination mit diversen Modulen, insbesondere jedoch Organic Groups. Dieses Modul erlaubt es, dass Teilnehmende der Plattform eigene Gruppen gründen und damit eigene soziale Relationen auf der Plattform erzeugen können. Viele Learning Management-Systeme verwenden den Kurs als zentrales sozialstrukturierendes Konzept und organisieren soziale Relationen häufig in Abhängigkeit zu Kursen. Die Dominanz des Kurses in der Gestaltung einer Lernumgebung erschwert jedoch das Gründen freier sozialer Strukturen unter den Teilnehmenden (vgl. Hölterhof und Kerres 2011). Die Fokussierung von Gruppen, die durch die Teilnehmenden frei gegründet werden können, soll dieser Dominanz entgegenwirken und den sozialen Austausch im Lernkontext fördern. Die Konzeption einer Lernumgebung als Soziales Netzwerk kann freiere soziale Strukturen realisieren, um die Dominanz des Kurses als sozialstrukturierendes Prinzip abzuschwächen und hierdurch Räume für eigene Kontakte und Beziehungen zu schaffen. Insbesondere wählbare und durch die Lernenden frei erstellbare Gruppen erscheinen hierbei interessant, um Kommunikationsprozesse jenseits von Kursen und Lehrangeboten zu ermöglichen. 
Die in der Plattform implementierten Inhalte sowie deren soziale Eingebundenheit in Lernprozesse stellen mögliche Gegenstände der Begegnung dar. Viele Modelle für Online- und Blended-Learning stellen die Lerninhalte als wesentliche Elemente von Konzeption und Gestaltung der Online-Lernumgebungen heraus (vgl. Garrison und Kanuka 2004; Halverson et al. 2014; Kerres und Witt 2003). Doch betonen sie dabei weniger das transformative Potenzial, welches die Aneignung solcher Inhalte auch in Online-Umgebungen auszeichnen kann (vgl. Garrison und Kanuka 2004; Mezirow 2000). Indem sie als mögliche Gegenstände für Begegnungen verstanden werden, rückt die Rolle einer eigengeleiteten und engagierten Aneignung von Inhalten ins Zentrum der Konzeption. Insbesondere Web Based Trainings und interaktive Lerninhalte folgen häufig eng strukturierten Lernpfaden und bieten wenig Anreize für eigene Explorationen und Engagement. Solche Inhalte als Elemente eines Möglichkeitsraumes für Begegnung aufzufassen und zu gestalten bedeutet, diese Inhalte für soziale Lernerlebnisse und aktive Konstruktionsprozesse der Subjekte zu konzipieren. Daher setzen Lernaufgaben im «OnlineCampus» Anreize für eigene und soziale Konstruktionsprozesse. Sie regen eine persönliche Auseinandersetzung mit Themen etwa als Einzel- oder Gruppenarbeit an und weisen dabei oft Peer-Feedback-Elemente auf, die in den Online-Foren der Plattform diskutiert werden. Peer-Feedback kann ein mediendidaktisches Gestaltungselement darstellen, welches Kommunikations- und Kollaborationsprozesse anregt (vgl. Topping 1998, 2009).

Das Phänomen der Begegnung ist nach Bollnow ferner durch einen offenen und unstetigen Prozess charakterisiert. Inwieweit Momenten dieser Unstetigkeit im formalen und zielgerichteten Lehr- und Lernprozess der wissenschaftlichen Weiterbildung tatsächlich eine angemessene Bedeutung zugeschrieben wird, ist zum grossen Teil eine studienorganisatorische Rahmenbedingung, die die Konzeption einer Online-Plattform überschreitet. Hinzu kommt, dass zwischen Lernprozessen und transformatorischen Bildungsprozesse zwar Überschneidungen bestehen, aber durchaus auch 
Unterschiede festgestellt werden können (vgl. Iske und Meder 2010; Meder 2007a). Demnach ist dieses Charakteristikum am Beispiel einer Lernumgebung nur eingeschränkt zu verorten. Aber dennoch können Momente der Unstetigkeit etwa als Offenheit, Selbstbestimmung und Partizipation in einer medienpädagogischen Konzeption berücksichtigt werden (vgl. Hölterhof und Schiefner-Rohs 2014) und so ansatzweise auch im Rahmen der Gestaltung einer OnlineUmgebung integriert werden. Insofern kann die Gestaltung sozialer Strukturen durch die Teilnehmenden auf der Plattform bereits als partizipatives Moment gesehen werden, welches Selbstbestimmung und Offenheit in der Anpassung an die eigene Situation ermöglicht. Auch die Offenheit für eigene Kommentare und Reflexionen zu Inhalten und Themen können durch eine Online-Umgebung gestaltet und gefördert werden. Die Konzeption einer Online-Umgebung als Möglichkeitsraum für Begegnungen kann demnach als partizipative Umgebung spezifiziert werden, die im Kontext sozialer Strukturen sowohl soziale als auch thematische Erfahrungsmöglichkeiten anbietet, als auch die eigene soziale Wahl und die informelle Fortführung von Kommunikationsprozesse integriert.

Als medienpädagogischer Gestaltungsprozess stellt neben der Konzeption und der Umsetzung schliesslich auch die Analyse ein wesentliches Element eines gestaltungsorientierten Forschungsprozesses dar. Content Management-Systeme speichern ihren Zustand in einer Datenbank, die für eine solche Analyse der OnlineUmgebungen herangezogen werden kann. Die Praxis zeigt jedoch, dass für eine solche Analyse die Rohdaten dieser Datenbank hypothesengeleitet aufbereitet werden müssen, um sie sodann in Forschungs- und Analysesoftware importieren zu können. Es muss eine Befehlssequenz entwickelt werden, welche die Rohdaten aus den unterschiedlichen Tabellen der Datenbank kombiniert und zusammenfügt. So speichert beispielsweise das Drupal-Modul Organic Groups die Zugehörigkeit der Nutzenden zu Gruppen in mehreren Datenbanktabellen, aus denen zunächst die erforderlichen Daten extrahiert werden müssen. Es bieten sich auch hier modulare und 
programmierbare Systeme an, da diese auf die Spezifika der eingesetzten Frameworks angepasst werden können. Ein Export der Datenbank erlaubt die Rekonstruktion auch der sozialen Strukturen der Plattform zu einem gewissen Zeitpunkt, etwa im Rahmen einer sozialen Netzwerkanalyse. Programmierbare Forschungswerkzeuge bieten darüber hinaus die Möglichkeit, die Datenbank ohne Export direkt anzusprechen. Die wissenschaftliche und statistische Analysesoftware R besitzt Funktionen, mit denen direkt Rohdaten vom Datenbankserver abgerufen werden können.

\section{Zusammenfassung}

Der Beitrag stellt Ansätze für die Gestaltung einer Online-Plattform als Soziales Netzwerk vor dem Hintergrund eines existenziellen Phänomens der Begegnung vor. Otto F. Bollnow deckt im Rahmen seiner existenziellen Pädagogik die Begegnung als Form unstetiger Bildung auf, welche auf spontane, erschütternde und unvorhersehbare Momente verweist, die zu Engagement und Transformation führen können (vgl. Bollnow 1977). Für die so aufgezeigte anthropologische und existenzielle Bedeutung von Begegnungen sind auch Kontingenz- und Fremdheitserfahrungen charakteristisch. Eine solche Konkretisierung bildender Begegnungen fokussiert ergebnisoffene und partizipative Erfahrungsräume, die mit dem persönlichen Engagement der Teilnehmenden einhergehen. Diese Charakterisierung des Begegnungsphänomens lässt Eigenheiten erkennen, die im Hinblick auf Soziale Netzwerke im Internet interessant erscheinen. Dabei sind Soziale Netzwerke im Internet Plattformen oder Umgebungen, in denen soziale Relationen zwischen den Teilnehmenden abgebildet werden. Sie zeichnen sich durch eine hohe Personalisierung aus, etwa indem Teilnehmende ein eigenes Profil anlegen können und Verbindungen zu anderen Teilnehmenden aufbauen können. Als solche sozialen Plattformen fokussieren sie bestimmte Themen oder Aktivitäten und repräsentieren Gegenstände, die für mögliche Begegnungen relevant werden können. Eine für Lern- und Bildungsprozesse relevante Funktion von sozialen Netzwerken im 
Internet besteht etwa in der Vernetzung der Teilnehmenden durch Kommunikations- und Kollaborationsprozesse, in denen Eindrücke mit und Erlebnisse von Lerninhalten und anderen Teilnehmenden ermöglicht werden. Insbesondere in der sozialen Eingebundenheit ihrer Inhalte strukturieren sie Begegnungserfahrungen und können somit als Möglichkeitsraum für Begegnungen interpretiert werden.

Medienpädagogische Online-Forschung als gestaltungsorientierte Bildungsforschung fokussiert die Konzeption, prototypische Umsetzung und Evaluation von Online-Umgebungen. Auf diese Weise werden auf der Grundlage einer medienpädagogischen Konzeption Erlebnisräume im Internet gestaltet und entwickelt. Eine Erörterung der Begegnung als existenziellem Phänomen kann für diese Gestaltungsprozesse fruchtbar gemacht werden, indem Online-Umgebungen als Möglichkeitsräume für Begegnungen gestaltet werden. Am Beispiel der Lernumgebung "OnlineCampus», die für weiterbildende Studienprogramme an der Universität DuisburgEssen entwickelt und eingesetzt wird, werden solche konzeptionellen Aspekte erörtert und Ansätze ihrer Realisierung durch Webtechnologie diskutiert. Es stellt sich heraus, dass der Einsatz eines Content Management-Systemes als Framework für die Implementierung eines Sozialen Netzwerks ein Kompromiss zwischen Komplexität der Programmierung und Gestaltungsfreiraum der Konzeption darstellt. So können für Lernplattformen typische Lerninhalte und Lernmaterialien wie etwa Texte, Video oder Web Based Trainings eingebettet werden in soziale Erfahrungsräume, die stärker eigengeleitete und forschende Lernprozesse fokussieren. Ferner ist es möglich, den Teilnehmenden der Plattform anhand frei wählbarer Gruppen, eigener Profile sowie anderen Partizipations- und Kollaborationsmöglichkeiten einen selbststrukturierten Erfahrungsraum zur Verfügung zu stellen, der weniger durch die Dominanz einer Kurs- oder Modulstruktur geprägt ist. 
In diesen und ähnlichen Aspekten verfolgt die Gestaltung der als Soziales Netzwerk realisierten Online-Umgebung die Konkretisierung eines Möglichkeitsraums für Begegnung als existenzielles und anthropologisches Phänomen. In der Fokussierung ihrer existenziellen Bedeutung überschreitet eine solche phänomenologische Erörterung des Lernens im Internet viele Modelle des Blended Learning und verortet Lernprozesse vor dem Hintergrund transformatorischer Bildungsprozesse.

\section{Literatur}

Akker, Jan Van den, Koeno Gravemeijer, Susan McKenney, und Nienke Nieveen. 2006. Educational Design Research. New York: Routledge.

Bollnow, Otto Friedrich. 1977. Existenzphilosophie und Pädagogik: Versuch über unstetige Formen der Erziehung. 5. Aufl. Stuttgart [u.a.]: Kohlhammer.

boyd, Danah M., und Nicole B. Ellison. 2007. «Social Network Sites: Definition, History, and Scholarship». Journal of Computer-Mediated Communication 13 (1): 210-30. https://doi.org/10.1111/j.10836101.2007.00393.x.

Easterday, Matthew W., Daniel R. Lewis, und Elizabeth M. Gerber. 2014. «Design-based research process: Problems, phases, and applications». Learning and Becoming in Practice, 1:317-24. https://www.isls. org/icls/2014/Proceedings.html.

Freeman, Linton C. 1978. «Centrality in Social Networks Conceptual Clarification». Social Networks I (3): 215-39. https://doi.org/10.1016/03788733(78)90021-7.

Garrison, D. Randy, und Heather Kanuka. 2004. «Blended Learning: Uncovering Its Transformative Potential in Higher Education». The Internet and Higher Education 7 (2): 95-105. https://doi.org/10.1016/j. iheduc.2004.02.001.

Halverson, Lisa R., Charles R. Graham, Kristian J. Spring, Jeffery S. Drysdale, und Curtis R. Henrie. 2014. "A Thematic Analysis of the Most Highly Cited Scholarship in the First Decade of Blended Learning Research». The Internet and Higher Education 20: 20-34. https://doi. org/10.1016/j.iheduc.2013.09.004. 
Heinen, Richard, Tobias Hölterhof, Michael Kerres, und Annabell Preussler. 2014. «Expertise: Analyse des Status-Quo und Empfehlungen für die Weiterentwicklung der religionspädagogischen Internet-Plattform〈Rpi-Virtuell〉». (Expertise im Auftrag des Comenius-Instituts). https://learninglab.uni-due.de/publikationen/3521.

Hitzler, Ronald. 1982. «Den Gegen-Stand verstehen: Zur Idee des Individuellen in der Sozialwissenschaft». Soziale Welt 33 (2): 136-56.

Hölterhof, Tobias. 2017a. «Die existenzielle Wende. Impulse einer philosophischen Idee für die Medienpädagogik». In Wenden: Interdisziplinäre Perspektiven auf das Phänomen turn, herausgegeben von Sandra Aßmann, Peter Moormann, Karina Nimmerfall, und Mirjam Thomann, 201-17. Wiesbaden: Springer Fachmedien. https://oi. org/10.1007/978-3-658-14805-8_17.

Hölterhof, Tobias. 2017b. «Sören Kierkegaard und das Internet: Partizipation und Engagement als Hinweis auf Medienbildung». In Medienimpulse: Beiträge zur Medienpädagogik 2014-2015, herausgegeben von Alessandro Barberi, Thomas Ballhausen, Christian Berger, Petra Missomelius, Katharina Kaiser-Müller, Ruth Sonderegger, Christian Swertz, und Christine W. Trültzsch-Wijnen, 341-47. Wien: new academic press.

Hölterhof, Tobias. 2018a. «Digitale Optionen für agile und unstetige Bildungsprozesse: Gestaltung einer sozialen Lernumgebung für die Hochschullehre». In Digitalisierung und Hochschulentwicklung: Proceedings zur 26. Tagung der Gesellschaft für Medien in der Wissenschaft e.V., herausgegeben von Barbara Getto, Patrick Hintze, und Michael Kerres, 106-18. Münster New York: Waxmann.

Hölterhof, Tobias. 2018b. «Soziale Netzwerkanalyse von Lernnetzwerken im Internet». In Netzwerk als neues Paradigma? Interdisziplinäre Zugänge zu Netzwerktheorien, herausgegeben von Florian Kiefer und Jens Holze, 83-100. Wiesbaden: Springer Fachmedien. https://oi. org/10.1007/978-3-658-18003-4_5.

Hölterhof, Tobias, und Michael Kerres. 2011. «Modellierung sozialer Kommunikation als Communities in Social Software und Lernplattformen». In INFORMATIK 2011: Informatik schafft Communities, herausgegeben von Hans-Ulrich Heiß, Peter Pepper, Holger Schlingloff, und Jörg Schneider, 434. Bonn: Köllen (Lecture Notes in Informatics). 
Hölterhof, Tobias, und Mandy Schiefner-Rohs. 2014. «Partizipation durch Peer-Education: Selbstbestimmung und Unstetigkeit in schulischen (Medien-)Bildungsprozessen». In Partizipative Medienkulturen: Positionen und Untersuchungen zu veränderten Formen öffentlicher Teilhabe, herausgegeben von Ralf Biermann, Johannes Fromme, und Dan Verständig, 283-99. Wiesbaden: Springer Fachmedien. https://oi. org/10.1007/978-3-658-01793-4_13.

Iske, Stefan, und Norbert Meder. 2010. «Lernprozesse als Performanz von Bildung in den Neuen Medien». In Digitale Lernwelten: Konzepte, Beispiele und Perspektiven, herausgegeben von Kai-Uwe Hugger und Markus Walber, 21-37. Wiesbaden: VS Verlag für Sozialwissenschaften. https://doi.org/10.1007/978-3-531-92365-9_2.

Jörissen, Benjamin, und Winfried Marotzki. 2009. Medienbildung - eine Einführung: Theorie - Methoden - Analysen. Bad Heilbrunn: Klinkhardt.

Kerres, Michael, Tobias Hölterhof, und Axel Nattland. 2011. «Zur didaktischen Konzeption von 〈Sozialen Lernplattformen〉 für das Lernen in Gemeinschaften». MedienPädagogik: Zeitschrift für Theorie und Praxis der Medienbildung, 1-22. https://doi.org/10.21240/ mpaed/oo/2011.12.09.X.

Kerres, Michael, Tobias Hölterhof, und Martin Rehm. 2016. «Lebenslanges Lernen im Kontext sozialer Medien: Chancen für formelles und informelles Lernen». In Lebenslanges Lernen im sozialstrukturellen Wandel: Ambivalenzen der Gestaltung von Berufsbiografien in der Moderne, herausgegeben von Dieter Münk und Marcel Walter, 141-70. Wiesbaden: Springer Fachmedien. https://doi.org/10.1007/978-3-65814355-8_8.

Kerres, Michael, und Martin Rehm. 2015. «Soziales Lernen im Internet Plattformen für das Teilen von Wissen in informellen und formellen Lernkontexten». HMD Praxis der Wirtschaftsinformatik 52 (1): 33-45. https://doi.org/10.1365/s40702-014-0112-2.

Kerres, Michael, und Claudia De Witt. 2003. «A Didactical Framework for the Design of Blended Learning Arrangements». Journal of Educational Media 28 (2-3): 101-13. https://doi.org/10.1080/13581650320 00165653 .

Kerres, Michael, und Claudia de Witt. 2011. «Zur (Neu)Positionierung der Mediendidaktik. Handlungs- und Gestaltungsorientierung in der Medienpädagogik». MedienPädagogik: Zeitschrift für Theorie und Praxis der Medienbildung 20: 259-70. https://doi.org/10.21240/ mpaed/20/2011.09.23.X. 
Koller, Hans-Christoph. 2011. «Anders werden. Zur Erforschung transformatorischer Bildungsprozesse». In Orte des Empirischen in der Bildungstheorie, herausgegeben von Ines Maria Breinbauer und Gabriele Weiß, 108-23. Würzburg: Königshausen \& Neumann.

Koskela, Jani. 2012. Discontinuity as Theoretical Foundation to Pedagogy. Existential Phenomenology in Otto Friedrich Bollnow's Philosophy of Education. Tampere: University of Oulu.

Meder, Norbert. 2007a. «Der Lernprozess als performante Korrelation von Einzelnem und kultureller Welt. Eine bildungstheoretische Explikation des Begriffs». Spektrum Freizeit, 119-35.

Meder, Norbert. 2007b. «Theorie der Medienbildung. Selbstverständnis und Standortbestimmung der Medienpädagogik». In Jahrbuch Medien-Pädagogik 6: Medienpädagogik - Standortbestimmung einer erziehungswissenschaftlichen Disziplin, herausgegeben von Werner Sesink, Michael Kerres, und Heinz Moser, 55-73. Wiesbaden: VS Verlag für Sozialwissenschaften. https://doi.org/10.1007/978-3-531-90544-0_3.

Mezirow, Jack. 2000. Learning as transformation: critical perspectives on a theory in progress. San Francisco: Jossey-Bass.

Pietraß, Manuela. 2009. «Medienbildung». In Handbuch Bildungsforschung, herausgegeben von Rudolf Tippelt und Bernhard Schmidt, 3. Aufl., 499-512. Wiesbaden: VS Verlag für Sozialwissenschaften. https://doi.org/10.1007/978-3-531-91831-0_26.

Röll, Franz Josef. 2010. «Social Network Sites». In Digitale Jugendkulturen, herausgegeben von Kai-Uwe Hugger, 209-24. Wiesbaden: VS Verlag für Sozialwissenschaften. https://doi.org/10.1007/978-3-53191908-9_12.

Rürup, Matthias, Heinke Röbken, Marcus Emmerich, und Imke Dunkake. 2015. Netzwerke im Bildungswesen: eine Einführung in ihre Analyse und Gestaltung. Wiesbaden: Springer VS.

Schweizer, Thomas. 1996. Muster sozialer Ordnung: Netzwerkanalyse als Fundament der Sozialethnologie. Berlin: D. Reimer.

Spanhel, Dieter. 2014. «Der Prozess der Medienbildung auf der Grundlage von Entwicklung, Lernen und Erziehung». In Perspektiven der Medienbildung, herausgegeben von Winfried Marotzki und Norbert Meder, 121-48. Wiesbaden: Springer Fachmedien Wiesbaden. https:// doi.org/10.1007/978-3-658-03529-7_6.

Topping, Keith. 1998. «Peer Assessment Between Students in Colleges and Universities». Review of Educational Research 68 (3): 249-76. https://doi.org/10.3102/00346543068003249. 
Topping, Keith J. 2009. «Peer Assessment». Theory Into Practice 48 (1): 20-27. https://doi.org/10.1080/00405840802577569.

Tulodziecki, Gerhard, Silke Grafe, und Bardo Herzig. 2013. Gestaltungsorientierte Bildungsforschung und Didaktik: Theorie - Empirie - Praxis. Bad Heilbrunn: Klinkhardt.

Tulodziecki, Gerhard, Bardo Herzig, und Silke Grafe. 2014. «Medienpädagogische Forschung als gestaltungsorientierte Bildungsforschung vor dem Hintergrund praxis- und theorierelevanter Forschungsansätze in der Erziehungswissenschaft». MedienPädagogik: Zeitschrift für Theorie und Praxis der Medienbildung, 1-18. https://doi.org/10.21240/ mpaed/oo/2014.03.10.X.

Walther, Joseph B. 1992. «Interpersonal Effects in Computer-Mediated Interaction: A Relational Perspective». Communication Research 19 (1): 52-90. https://doi.org/10.1177/009365092019001003.

Wasserman, Stanley, und Katherine Faust. 1994. Social network analysis: methods and applications. Cambridge, New York: Cambridge University Press. 